\title{
Estimating Impervious Surface Distribution: A Comparison of Object Based Analysis and Spectral Mixture Analysis
}

\author{
Chunzhu WEI and Thomas BLASCHKE \\ University of Salzburg / Austria ·weichunzhuzhu@gmail.com \\ This contribution was double-blind reviewed as extended abstract.
}

\begin{abstract}
Knowledge about the distribution of impervious surface is important to the understanding of urbanization processes and environmental consequences. This paper compares two methods for classifying the degree of imperviousness for the large metropolitan region of the fast growing city of Guangzhou in southern China: spectral mixture analysis (SMA) and object based image analysis (OBIA). Both methods were applied to data from the Chinese Satellite Huan Jing 1-B (HJ-1B). Samples were classified using a Support Vector Machine (SVM), ultimately yielding the percentages of Impervious Surface Areas (ISA). The estimation accuracy for ISA was assessed using the land surface temperature retrieval result based on HJ-1B satellite data. In general, OBIA achieved slightly better results, while both methods achieved low accuracies in the highly vegetated northern and eastern parts of the study area. Our results show that OBIA combined with SVM classification of the HJ-1B data provides a means to map and quantify the degree of impervious surface distribution.
\end{abstract}

\section{Introduction}

Impervious surfaces are anthropogenic features through which water and air cannot infiltrate the soil, including, for example, roads, parking lots, and buildings. Research on impervious surface extraction from remotely sensed data has attracted interest since the late 1970s (Lu \& Weng 2006). For three decades, remotely sensed imagery has been used for urban change detections (Lu \& Weng 2006, Weng 2012). Many applications do not only require information about the amount of sealed surfaces and statistical tables, but also detailed maps. Accurate measurement of the distribution of impervious surface areas can provide an essential indicator for environmental quality analysis (Arnold $\mathrm{Jr}$ \&Gibbons 1996, Dougherty et al. 2004). Percent of impervious surface area has emerged as a key factor to explain and generally predict the degree of the corresponding decrease infiltration to streams and watersheds. Arnold Jr and Gibbons (1996) suggest impervious surface area as a measure of land use that is closely correlated with the negative impacts of sealing, and more generally that the amount of impervious surface in a landscape is an important indicator of environmental and habitat quality in urban areas. For urban climate, Yuan and Bauer (2006) have documented a strong relationship between the amount of impervious surface area and land surface temperatures, or the urban heat island effect. Subsequently, 
impervious surface information is fundamental for watershed planning and management and for urban planning and policy making (Bauer et al. 2008).

Various techniques, including traditional ground surveys with GPS and aerial photography interpretation have been used to characterize and quantify impervious surfaces areas. However, field work and aerial photography interpretation are time-consuming and sometimes subjective. In this paper we will therefore focus on satellite remote sensing data which is widely available today and suitable for monitoring purposes. Spatial resolution is the most obvious parameter when choosing remote sensing data for Impervious Surface Areas (ISA) estimates. Since mixed pixels have generally been recognized as a problem affecting the effective use of remotely sensed data in urban land-use/cover classification (Cracknell 1998), scientists tend to use the highest spatial resolutions possible. The most widely used sensor in this respect is Landsat, with its $30 \mathrm{~m}$ resolution for TM and ETM+. The Chinese HJ-1B satellite has similar characteristics but is nearly exclusively used by Chinese scientists and for China.

For medium data spatial resolution $(10-100 \mathrm{~m})$, traditional per-pixel classifiers, such as the maximum-likelihood classifier, have limitations for complex urban landscapes due to the mixed-pixel problem, which in principle applies to any resolution. One of the major advances in urban land-use/cover analysis is Ridd's (1995) vegetation-impervious surfacesoil (V-I-S) model. This model assumes that the spectral signature of land cover in urban environments is a linear combination of three components, namely vegetation, impervious surface, and soil. The V-I-S model has demonstrated to be an effective approach to coping with the mixed-pixel problem, as well as providing a guideline for decomposing lowresolution images of urban landscapes, and a link for these components to spectral signatures (see Weng 2012). For medium spatial resolutions $(10-100 \mathrm{~m})$, classification algorithms such as spectral mixture, maximum likelihood, and decision trees analysis are widely used for identifying the impervious surfaces area (Jennings et al. 2004). However, these image classification methods heavily depend on feature selection and may not be robust enough for a spectrally complex environment.

An alternative is the object based image analysis (OBIA) approach, which aims at objects and allows the combination of the characteristics shape, texture, context and relationship with neighbors into the image analysis. It has become popular in remote sensing applications and is increasingly used in landscape analysis (Blaschke 2010). It is even claimed to be a paradigm in remote sensing (Blaschke et al. 2014). In this study we compare spectral mixture analysis (SMA) and OBIA, and use support vector machine (SVM) classifiers for both approaches. SVM has been proposed to handle high dimensional data without over fitting the training data to the data margins (Mountrakis et al. 2011). In order to evaluate the accuracy of the two methods for ISA, this study quantifies the spatial and spectral characteristics of urban impervious layers and uses SVM to train the samples and to classify the pervious and impervious layers for the province of Guangzhou in China.

\section{Methods}

\subsection{Study area and data processing}

The study area includes the fast growing Mega-city of Guangzhou with high intensities of economic activities causing serious urban environment problems, including suburban 
underlying surface anomalies. It also includes some vegetated areas, particularly in the northern and eastern parts. The total area is 7,434.4 square kilometers. HJ-1B data of 23th September 2009 was used. As pre-processing steps, radiometric calibration and atmospheric correction were carried out based on the HJ-1A/1B tools V3.0 and FLAASH atmospheric correction model in ENVI.

\subsection{Classification scheme and sample determination}

Impervious surface shows a correlation with land use. Firstly, we chose samples that represent distinct land use based on SMA and OBIA separately. Then, the impervious layers and pervious layers were classified by means of SVM. For sample determination in the SMA, we applied a Maximum Noise Fraction (MNF) as implemented in the software ENVI v 4.7 to build a statistical model for the images to transform the noise fraction in the data (Lee et al. 1999). MNF results were imported into 2D scatter plots. The endpoints in the $2 \mathrm{D}$ scatter plots were chosen as the samples of different land use types (including grassland, forest, farmland, rivers, built-up area and roads). For the OBIA part, we segmented the image into objects with the multi-resolution segmentation algorithm using a scale parameter of 50 in eCognition software $v$ 8.9. The nearest neighbor classification was applied to extract the same sample types in SMA). Several fuzzy rule-based parameters like Normalized Difference Vegetation Index (NDVI), Land and Water Masks (LWM) and Specific Leaf Area Vegetation Index (SLAVI) were added to different samples to prepare for the next step (Benz et al. 2004).

\subsection{ISA retrieval based on SVM}

The samples that were extracted above were divided into two main types: impervious layer (mainly built-up areas and roads) and pervious layer (including the grassland, forest, farmland, rivers). These samples were exported to text files and normalized. The kernel functions-radial basis function (RBF) of svm.train in LIBSVM was used to train the samples into a high dimensional training space. Then we used the easy.py, grid.py and gnuplot functions of Libsvm to calculate the optimized parameters $\mathrm{C}$ (error tolerance) and gamma (data in high dimension distribution) (Chang \& Lin 2011). Finally we imported C and gamma into ENVI-SVM and eCognition-SVM classifiers respectively, in order to apply SVM for pervious and impervious feature classification in high dimensional space. We used the values $\mathrm{C}=2$ and gamma $=0.25$ for $\mathrm{ENVI}$, and $\mathrm{C}=3$, gamma $=0.33$ for eCognition.

\section{Results}

In addition to the samples that were used to analyze ISA in ENVI and eCognition, 20 other samples of pervious and impervious features were used for the assessment of both ISA classifications. The accuracy of land use retrieval from SMA and OBIA (shown in Figure 1) were $72 \%$ and $78 \%$, respectively. Large areas of ISA are in conformity with the urban development concentrated in the south-eastern part of the province, which is the Central Business District of Guangzhou. However, the SMA retrieval result is poor for the forest and grassland areas. In order to evaluate the ISA results further, land surface temperature (LST) was retrieved from the thermal infrared band of HJ-1B using the JM\&S algorithm 
(Gao, Sun, et al. 2010). High temperature areas should correlate with ISA. Normalization was used to reclassify LST into three types: High, Normal and Low. The percentage of ISA and the corresponding average values for the three LST types were calculated. Table 1 shows that the OBIA classification results were closer to the LST values for Guangzhou.

Tab. 1: Percent of ISA in various LST classes

\begin{tabular}{|l|l|l|l|l|l|}
\hline & Years & \%ISA & High & Normal & Low \\
\hline SMA & 2009 & $7.2 \%$ & $8.6 \%$ & $71.7 \%$ & $24.6 \%$ \\
\hline OBIA & 2009 & $31.84 \%$ & $14.69 \%$ & $73.06 \%$ & $12.25 \%$ \\
\hline
\end{tabular}

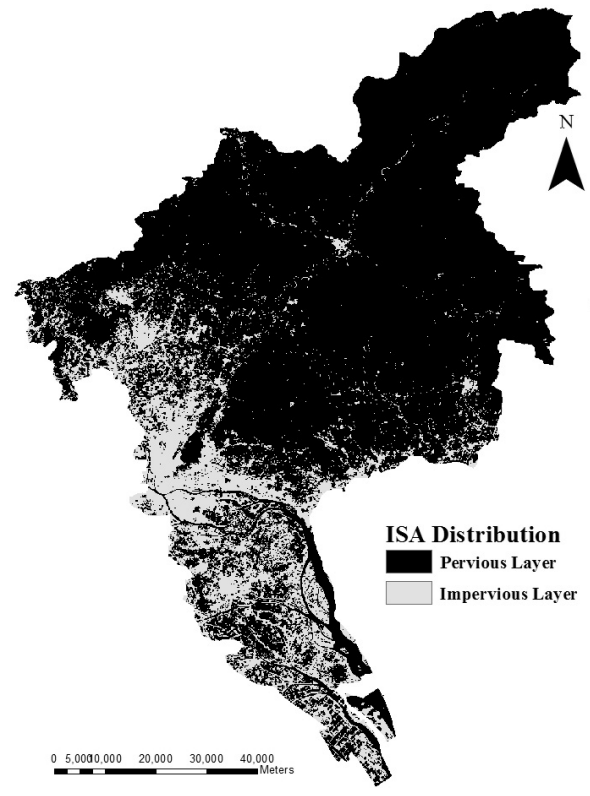

(a)

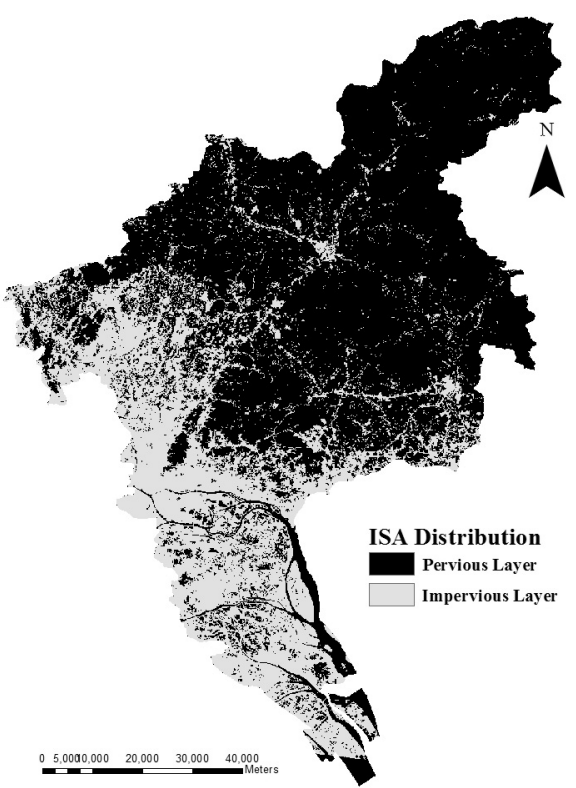

(b)

Fig. 1: Comparison of ISA: (a) ISA based on SMA and SVM; (b) ISA based on OBIA and SVM

\section{Conclusion}

We can conclude that data from the Landsat-like Chinese HJ-1B allowed the mapping of impervious surface area analysis in a reasonable spatial resolution. When comparing SMA and OBIA, the latter resulted in a more accurate impervious surface map. SMA is a purely spectral approach and does not allow the utilization of neighborhood relations. In accordance with Lu and Weng (2006) we can confirm that the extraction of impervious surfaces is still challenging because of the complexity of urban and suburban landscapes, particularly with medium spatial resolutions. In our study, both classification methods for ISA achieved poor results for highly vegetated areas. In such situations, impervious 
surfaces may be partially under vegetation cover or may be mixed with other land cover types, such as trees or bushes.

\section{References}

ARnold J., Chester L. \& JAMES GiBbOns, C. (1996), Impervious surface coverage: the emergence of a key environmental indicator. Journal of the American Planning Association, 62 (2), 243-258.

Bauer, M. E., Loffelholz, B. C. \& Wilson, B. (2008), Estimating and mapping impervious surface area by regression analysis of Landsat imagery (pp. 3-19). CRC Press: Boca Raton.

BLASCHKE, T. (2010), Object based image analysis for remote sensing. ISPRS Journal of Photogrammetry and Remote Sensing, 65 (1), 2-16.

BlaschKe, T., Hay, G. J., Kelly, M. et al. (2014), Geographic Object-Based Image Analysis - Towards a new paradigm. ISPRS Journal of Photogrammetry and Remote Sensing, 87, 180-191.

Benz, U. C., Hofmann, P., Willhauck, G., Lingenfelder, I., \& Heynen, M. (2004), Multi-resolution, object-oriented fuzzy analysis of remote sensing data for GIS-ready information. ISPRS Journal of photogrammetry and remote sensing, 58 (3), 239-258.

ChAng, C. C. \& LIN, C. J. (2011), LIBSVM: a library for support vector machines. ACM Transactions on Intelligent Systems and Technology, 27 (2), 1-27.

CRACKNEll, A. P. (1998), Synergy in remote sensing - What's in a pixel? International Journal of Remote Sensing, 19, 2025-2047.

DougherTy, M. et al. (2004), Evaluation of impervious surface estimates in a rapidly urbanizing watershed. Photogrammetric Engineering and Remote Sensing, 70 (11), 1275-1284.

GaO, T., Sun, L., Xu, J. \& HAN, L. (2010), Study on Urban Heat Island effect of Beijing based on HJ-1B IRS data. In Information Science and Engineering (ICISE), 2010 2nd International Conference on, IEEE, 6975-6978.

Jennings, D. B., Jarnagin, S. T. \& EBert, D. W. (2004), A modeling approach for estimating watershed impervious surface area from National Land Cover Data. Photogrammetric Engineering and Remote Sensing, 70 (11), 1295-1307.

LU, D. \& WENG, Q. (2006), Use of impervious surface in urban land-use classification. Remote Sensing of Environment, 102 (1), 146-160.

Lee, J. B., Woodyatt, A. S. \& Berman, M. (1990), Enhancement of high spectral resolution remote-sensing data by a noise-adjusted principal components transform. Geoscience and Remote Sensing, 28 (3), 295-304.

Mountrakis, G., IM, J. \& OGOLE, C. (2011), Support vector machines in remote sensing: A review. ISPRS Journal of Photogrammetry and Remote Sensing, 66 (3), 247-259.

RIDD, M. K. (1995), Exploring a V-I-S (Vegetation-Impervious Surface-Soil) model for urban ecosystem analysis through remote sensing: Comparative anatomy for cities. International Journal of Remote Sensing, 16, 2165-2185.

WENG, Q. (2012), Remote sensing of impervious surfaces in the urban areas: Requirements, methods, and trends. Remote Sensing of Environment, 117, 34-49.

YUAN, F. \& BAUER, M. E. (2006), Comparison of impervious surface area and normalized difference vegetation index as indicators of surface urban heat island effects in Landsat imagery. Remote Sensing of Environment, 106 (3), 375-386. 